Хронический аспергиллез легких: обзор литературы и демонстрация собственных наблюдений

\author{
Николаева Н.Г., Шадривова О.В., Ицкович И.Э., Климко Н.Н. \\ ФГБОУ ВО «Северо-Западный государственный медицинский университет им. И.И. Мечникова» Минздрава России, \\ ул. Кирочная, 41, Санкт-Петербург, 191015, Российская Федерация
}

Николаева Наталия Георгиевна, аспирант кафедры лучевой диагностики и лучевой терапии, врач-рентгенолог кабинета компьютерной томографии клиники им. Э.Э. Эйхвальда ФГБОУ ВО «Северо-Западный государственный медицинский университет им. И.И. Мечникова» Минздрава России;

https://orcid.org/0000-0001-7211-892X

Шадривова Ольга Витальевна, к. М. Н., доцент кафедры клинической микологии, аллергологии и иммунологии ФГБОУ ВО «Северо-Западный государственный медицинский университет им. И.И. Мечникова» Минздрава России;

https://orcid.org/0000-0002-3272-5263

Ицкович Ирина Эммануиловна, д. М. н., профессор, заведующая кафедрой лучевой диагностики и лучевой терапии, заведующая рентгеновским отделением клиники им. Э.Э. Эйхвальда ФГБОУ ВО «Северо-Западный государственный медицинский университет им. И.И. Мечникова» Минздрава России;

https://orcid.org/0000-0001-8352-3955

Климко Николай Николаевич, д. М. Н., профессор, заведующий кафедрой клинической микологии, аллергологии и иммунологии ФГБОУ ВО «Северо-Западный государственный медицинский университет им. И.И. Мечникова» Минздрава России;

https://orcid.org/0000-0001-6095-7531

\begin{abstract}
Резюме
Хронический аспергиллез легких (ХАЛ) - тяжелое заболевание, развивающееся преимущественно основным инструментальным методом диагностики ХАЛ, необходимым для определения формы заболевания, выбора тактики лечения, контроля осложнений и мониторинга терапии. Это обусловливает чевые слова: computed tomography, chronic pulmonary aspergillosis, aspergilloma, air-crescent symptom, differential diagnosis. воздушного серпа; дифференциальная диагностика; обзор.

Конфликт интересов. Авторы заявляют об отсутствии конфликта интересов. логии. 2021; 102(5): 317-28. https://doi.org/10.20862/0042-4676-2021-102-5-317-328

Для корреспонденции: Николаева Наталия Георгиевна, E-mail: nikolasha85@list.ru

Статья поступила 13.01.2021

После доработки 13.04.2021

Принята к печати 14.04.2021

\section{Chronic Pulmonary Aspergillosis: Literature Review and Demonstration of Own Observations} у пациентов без выраженных нарушений иммунного статуса. Компьютерная томография является важность понимания рентгенологом основных аспектов своевременной и дифференциальной диагностики. Отечественных исследований по этой проблеме недостаточно. В данной работе проведен анализ российских и зарубежных публикаций за период 2014-2020 гг., представленных в электронных базах данных PubMed, Web of Science, Elsevier, eLibrary. При поиске информации использованы следующие клю-

Ключевые слова: хронический аспергиллез легких; компьютерная томография; аспергиллома; симптом

Для цитирования: Николаева Н.Г., Шадривова О.В., Ицкович И.Э., Климко Н.Н. Хронический аспергиллез легких: обзор литературы и демонстрация собственных наблюдений. Вестник рентгенологии и радио-

\author{
Natalia G. Nikolaeva, Olga V. Shadrivova, Irina E. Itskovich, Nikolay N. Klimko \\ Mechnikov North-West State Medical University, Ministry of Health of the Russian Federation, \\ ul. Kirochnaya, 41, Saint Petersburg, 191015, Russian Federation
}

Natalia G. Nikolaeva, Postgraduate, Chair of Radiology, Radiologist, Computed Tomography Room, Eichwald Clinic, Mechnikov NorthWest State Medical University;

https://orcid.org/0000-0001-7211-892X

Olga V. Shadrivova, Cand. Med. Sc., Associate Professor, Chair of Clinical Mycology, Allergology and Immunology, Mechnikov North-West State Medical University;

https://orcid.org/0000-0002-3272-5263 
Irina E. Itskovich, Dr. Med. Sc., Professor, Chief of Chair of Radiology; Head of X-Ray Department, Eichwald Clinic, Mechnikov North-West State Medical University;

https://orcid.org/0000-0001-8352-3955

Nikolay N. Klimko, Dr. Med. Sc. Professor, Chief of Chair of Clinical Mycology, Allergology and Immunology, Mechnikov North-West State Medical University;

https://orcid.org/0000-0001-6095-7531

\begin{abstract}
Chronic pulmonary aspergillosis (CPA) is a severe disease that develops mainly in patients without obvious immune disorders. Computed tomography is the main instrumental method in the diagnosis of CPA, which is necessary to determine the form of the disease, to choose treatment policy, to combat complications, and to monitor therapy. This makes it important for a radiologist to understand the main aspects of timely and differential diagnosis. There are insufficient Russian studies on this problem. This paper analyzes the 2014-2020 Russian and foreign publications available in PubMed, Web of Science, Elsevier, and eLibrary electronic databases. When searching for information, the following keywords were used: "computed tomography", "chronic pulmonary aspergillosis", "aspergilloma", "air-crescent symptom", "differential diagnosis".
\end{abstract}

Keywords: chronic pulmonary aspergillosis; computed tomography; aspergilloma; air-crescent symptom; differential diagnosis; review.

Conflict of interest. The authors declare no conflict of interest.

For citation: Nikolaeva NG, Shadrivova OV, Itskovich IE, Klimko NN. Chronic pulmonary aspergillosis: literature review and demonstration of own observations. Journal of Radiology and Nuclear Medicine. 2021; 102(5): 317-28 (in Russian). https://doi.org/10.20862/0042-4676-2021-102-5-317-328

For corresponding: Natalia G. Nikolaeva, E-mail: nikolasha85@list.ru

\section{Введение}

В мире насчитывается около 3 млн больных хроническим аспергиллезом легких (ХАЛ), из них в Европе - около 240 тыс. [1]. Согласно методике подсчета, предложенной обществом LIFE (Leading International Fungal Education), общее число пациентов с ХАЛ в России составляет 52311 человек, показатель заболеваемости на 100 тыс. населения - 36 случаев [2]. Частота встречаемости ХАЛ в странах ближнего зарубежья (Украина, Таджикистан) варьирует: 22 и 46 случаев на 100 тыс. населения соответственно $[3,4]$. Однако данные о распространенности заболевания, особенно в развивающихся регионах, могут быть недооценены в связи с тем, что ХАЛ нередко диагностируют на поздних стадиях. Поздняя диагностика и отсутствие адекватного лечения при ХАЛ приводят к высокой летальности - до 50\% в течение 5 лет [1]. В исследовании D. Lowes et al. сообщается, что показатели 1-, 5- и 10-летней выживаемости составляют $86 \%, 62 \%$ и $47 \%$ соответственно [5].

Согласно данным того же исследования повышенный риск летальности при ХАЛ связан как с наличием фонового заболевания (нетуберкулезный микобактериоз, хроническая обструктивная болезнь легких (ХОБЛ), саркоидоз), так и с прогрессированием микотического процесса и его осложнениями: двусторонним поражением, легочным кровотечением, а также присоединением бактериальной инфекции [5].
В большинстве исследований установлено, что ХАЛ чаще возникает у лиц среднего и пожилого возраста, мужского пола, не имеющих выраженных нарушений иммунного статуса. В связи с этим у них может наблюдаться бессимптомное течение и нетипичные результаты лучевого обследования, что затрудняет раннюю диагностику [6-8].

В отечественной литературе обсуждаются ранние признаки инвазивного аспергиллеза легких, полученные при компьютерной томографии (КT) [8], а также КТ-картина в динамике заболевания [9].

Таким образом, актуальность анализа литературы определяется как возрастанием распространенности ХАЛ, так и нерешенными вопросами ранней диагностики и лечения.

\section{Этиология и патогенез ХАЛ}

Известно более 400 видов грибов рода Aspergillus [10]. Они широко распространены в окружающей среде [11]. Споры грибов рода Aspergillus в большом количестве можно обнаружить в строительном и сельскохозяйственном мусоре, сырых стенах, компосте, комнатных растениях. Исследования качества воздуха показали, что ежедневно человек может вдыхать сотни этих спор, но лишь у небольшого процента людей возникает инфицирование [12].

Наиболее частыми возбудителями ХАЛ у человека являются A. fumigatus, A. flavus, A. niger и A.terreus [11]. Известны клинические случаи ХАЛ, вызванные более редкими видами, такими 
как Aspergillus ochareus [13]. Гистологически мицелий Aspergillus представляет собой отдельные септированные дихотомически ветвящиеся под углом $45^{\circ}$ гифы. На гифах расположены конидиеносцы, которые содержат сотни конидий диаметром от 2 до 3 мм [14].

Клинико-рентгенологическая форма заболевания определяется взаимодействием между пациентом и Aspergillus, а также наличием или отсутствием повреждения легочной паренхимы [15].

Структурные изменения легочной ткани, такие как посттуберкулезные полости и каверны, бронхоэктазии, идиопатический легочный фиброз с формированием «сотового легкого», буллезная эмфизема, состояния после оперативных вмешательств, деструктивной пневмонии, абсцесса, могут предрасполагать к развитию хронического аспергиллеза [15]

\section{Методы и критерии диагностики ХАЛ}

В 2016 г. Европейским респираторным обществом (European Respiratory Society, ERS) совместно с Европейским обществом клинической микробиологии и инфекционных болезней (European Society of Clinical Microbiology and Infectious Diseases, ESCMID) сформулированы следующие диагностические критерии ХАЛ [1]: хроническое (более 3 мес) течение заболевания, стойкие изменения при КТ легких, выявление специфического иммуноглобулина $\mathrm{G}$ (Immunoglobulin $\mathrm{G}, \mathrm{IgG}$ ) или преципитинов к Aspergillus в сыворотке крови, выявление мицелия Aspergillus spp. в окрашенных мазках или биопсийном материале, выделение Aspergillus spp. при посеве биопсийного материала, бронхоальвеолярного лаважа (БАЛ), мокроты, а также исключение альтернативных заболеваний (при наличии более одной полости). Таким образом, КТ является обязательным условием диагностики ХАЛ. Ранняя диагностика ХАЛ необходима для успешного лечения, однако существуют определенные трудности в интерпретации КТ.

Согласно вышеуказанным клиническим рекомендациям выделяют следующие клинико-рентгенологические формы ХАЛ: одиночная аспергиллома, нодулярный аспергиллез, хронический кавернозный аспергиллез и фиброзирующий аспергиллез (рис. 1). Возможно сочетание КТ-признаков патологического процесса [1]. Необходимость дифференциации различных форм ХАЛ обусловлена тем, что диагностическая и лечебная тактика в разных случаях различается. Диагностические рекомендации [1] подчеркивают необходимость как лучевого, так и лабораторного подтверждения диагноза ХАЛ. Лабораторное подтверждение диагноза ХАЛ проводят на основании серологических, микробиологических (микроскопия и посев биоптата, мокроты, БАЛ и пр.) и гистологических методов исследования [14].
Определение в сыворотке крови специфичного для Aspergillus fumigatus IgG является ключевым диагностическим признаком ХАЛ $[1,16,17]$. Данные о чувствительности и специфичности определения Aspergillus-специфического IgG в сыворотке крови в литературе варьируют в пределах 80-90\% [16]. Для простой аспергилломы чувствительность и специфичность IgG к Aspergillus составляют 63,5\% и 98,3\% соответственно [18]. Специфический к Aspergillus IgМ показал более высокую чувствительность при полуинвазивном аспергиллезе и не имеет значения в диагностике ХАЛ [17]. Следует отметить, что у некоторых пациентов, в частности с простой одиночной аспергилломой или нодулярной формой ХАЛ, IgG к Aspergillus может оставаться отрицательным при явных клинических и лучевых проявлениях заболевания [19].

Галактоманнан является полисахаридным компонентом клеточной стенки грибов рода Aspergillus и высвобождается в окружающие ткани во время активного роста или инвазии [20]. Определение галактоманнана в сыворотке крови характеризуется низкой чувствительностью и не имеет ценности для диагностики ХАЛ, в отличие от инвазивного аспергиллеза [19, 21]. Обнаружение антигена галактоманнана в бронхоальвеолярном лаваже у пациентов с ХАЛ является более информативным: чувствительность и специфичность составляют $68 \%$ и 93\% соответственно при пороговом значении индекса оптической плотности более 0,5 [20].

У пациентов с ХАЛ часто повышены общие маркеры воспаления, однако это не является решающим в диагностике [1]

Для идентификации возбудителя используют культуральные методы диагностики. Выделение чистой культуры возбудителя дает возможность определить его чувствительность к лекарственным препаратам [10].

Помимо лабораторных методов немалую роль в дифференциальной диагностике играет гистологическое исследование, которое позволяет исключить злокачественные новообразования и другие заболевания со сходными проявлениями. Наличие септированного мицелия, дихотомически ветвящегося под острым углом, без инвазии легочной паренхимы указывает на ХАЛ [11].

Таким образом, комбинация лабораторных методов повышает чувствительность диагностики ХАЛ.

\section{Основные фоновые заболевания и клинические проявления ХАЛ}

\section{Туберкулез}

Туберкулез легких является основным фактором риска развития ХАЛ вследствие наличия остаточных полостей в паренхиме [21]. Терапия 

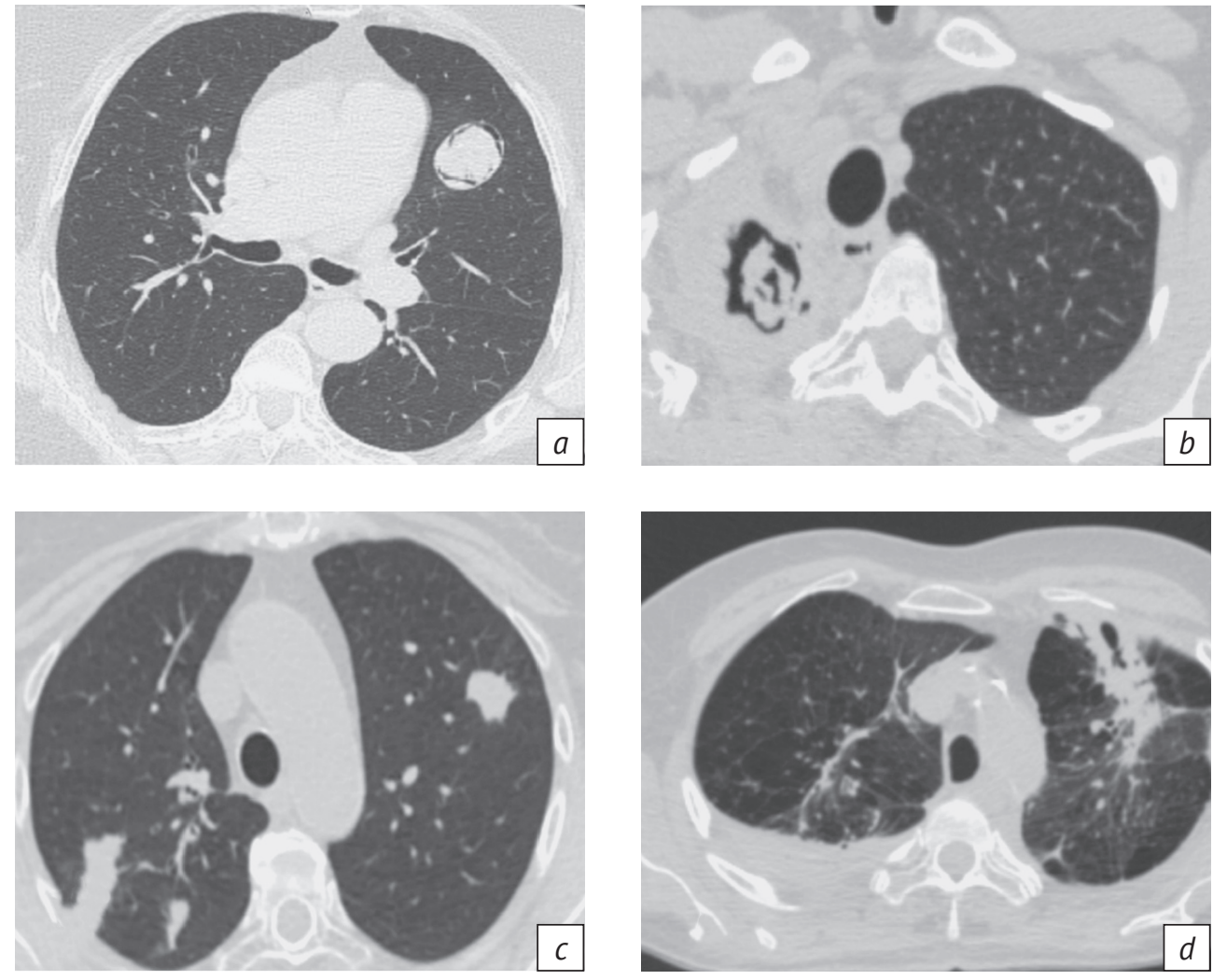

Puc. 1. Компьютерные томограммы различных вариантов ХАЛ (легочное окно, аксиальная проекция):

$a$ - пациентка К., 64 года: простая одиночная аспергиллома (полость в легком, содержащая грибной шар); жалоб нет, образование в легком выявлено при плановой рентгенографии, основное заболевание не установлено; по результатам исследования бронхоальвеолярного лаважа, антиген к Aspergillus spp. положительный, индекс оптической плотности 2,3; показано оперативное лечение на фоне приема антимикотических препаратов; $b$ - пациентка С., 47 лет: хронический кавитарный легочный аспергиллез (крупная полость в верхушке правого легкого, содержащая грибной шар); выраженное утолщение паракостальной плевры; основное заболевание - кавернозный туберкулез (МБТ-); показано оперативное лечение на фоне приема антимикотических препаратов; с - пациентка М., 65 лет: нодулярный аспергиллез (множественные двусторонние узловые образования в легких); основное заболевание - бронхиальная астма, принимает ингаляционные глюкокортикостероиды; жалобы на кашель с отхождением мокроты слизистого характера; по результатам анализа крови, специфический IgG к Aspergillus fumigatus 1:400 (норма 1:100); при посеве мокроты получен рост A. fumigatus в двух точках посева; назначена антимикотическая терапия; $d$ - пациентка А., 58 лет: фиброзирующий легочный аспергиллез (двусторонние изменения: грубый фиброз в сочетании с полостями и утолщением костальной плевры); основное заболевание - саркоидоз легких; по результатам анализа крови, специфический IgG к Aspergillus fumigatus 1:800 (норма 1:100); показана антимикотическая терапия

Fig. 1. Computed tomography scans of different CPA types (pulmonary window, axial projection):

$a$ - female patient K. aged 64 years: simple single aspergilloma (the pulmonary cavity containing a fungus ball); no complaints; a lung mass has been identified on routine radiography; no underlying disease has been identified; examination of bronchoalveolar lavage has shown a positive Aspergillus spp. antigen and an optical density index of 2.3. Surgical treatment is indicated while taking antifungal drugs; $b$ - female patient $\mathrm{S}$. aged 47 years: chronic cavitary pulmonary aspergillosis (a large cavity in the right lung apex, which contains a fungus ball); obvious paracostal pleural thickening; the underlying disease is cavernous tuberculosis (MBT-); surgical treatment is indicated while taking antifungal drugs; $c$ - female patient M. aged 65 years: nodular aspergillosis (multiple bilateral lung nodules); the underlying disease is asthma; she is taking inhaled glucocorticosteroids; complaints of cough with expectoration; a blood test has indicated Aspergillus fumigatus-specific IgG 1:400 (normal value, 1:100); sputum cultures have yielded a growth of $A$. fumigatus at two inoculation points; antifungal therapy is prescribed; $d$ - female patient A. aged 58 years: fibrosing pulmonary aspergillosis (bilateral changes: severe fibrosis concurrent with costal pleural cavities and thickening; the underlying disease is pulmonary sarcoidosis; a blood test has revealed Aspergillus fumigatus-specific IgG 1:800 (normal value, 1:100); antifungal therapy is recommended

данной группы пациентов требует тщательного подхода с учетом лекарственного взаимодействия антифунгальных и противотуберкулезных препаратов [10, 22]. Общие показатели заболеваемости ХАЛ после перенесенного тубер- кулеза могут быть недооценены: вследствие схожей клинической и лучевой картины ХАЛ ошибочно принимают за реактивацию туберкулеза, обусловленную резистентным возбудителем $[4,23]$. 

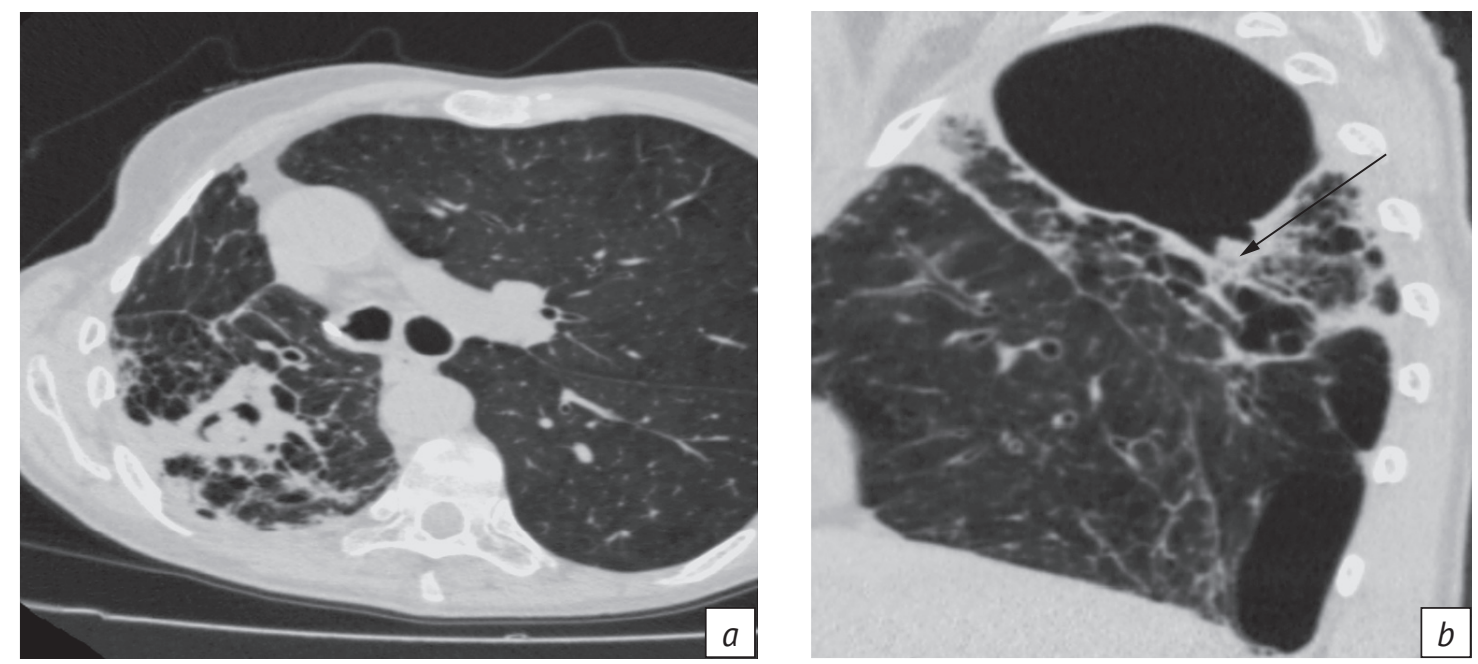

Puc. 2. Компьютерные томограммы пациентки Т., 64 года: легочное окно, аксиальная (a) и сагиттальная (b) проекции. Хронический аспергиллез легких (кавитарная форма). Состояние после правосторонней лобэктомии по поводу инфильтративного туберкулеза от 2015 г. В 2016 г. - ухудшение состояния (кашель, повышение температуры). Расценено как рецидив туберкулеза. Назначена противотуберкулезная терапия. В нижних отделах крупной полости определяется мягкотканный субстрат, окруженный серповидной полоской воздуха (указано стрелкой). В последующем - лабораторное подтверждение ХАЛ

Fig. 2. Computed tomography scans in female patient T. aged 64 years: pulmonary window, axial (a) and sagittal (b) projections. Chronic pulmonary aspergillosis (a cavitary type). Her condition after right-sided lobectomy for infiltrative tuberculosis was observed in 2015. In 2016, she had a deteriorated condition (cough, fever). This is regarded as a tuberculosis relapse. Antitubercular therapy was prescribed. The lower portions of the large cavity display a soft-tissue substrate surrounded by an air crescentshaped strip (arrow). CPA has been subsequently confirmed by laboratory tests

Изучение компьютерных томограмм пациентов, прошедших лечение от туберкулеза, показало наличие сохраняющихся полостей и бронхоэктазов как наиболее часто встречаемых последствий (рис. 2) $[23,24]$.

Риск развития микотической инфекции в посттуберкулезных полостях ежегодно составляет 6,5$32,7 \%[24,25]$.

В странах, эндемичных по туберкулезу, выявляется высокая заболеваемость ХАЛ, при этом существуют сложности диагностики ХАЛ в условиях ограниченных ресурсов [23, 26, 27]. Достоверной зависимости между формой туберкулезного процесса, режимом химиотерапии туберкулеза и развитием ХАЛ не выявлено [25].

КТ играет ключевую роль в мониторинге пациентов после перенесенного туберкулеза, оценке дальнейших изменений паренхимы легких и поздних осложнений, таких как ХАЛ. Диагностический алгоритм, включающий в себя выявление в сыворотке крови повышенного уровня IgG к Aspergillus, полостей в легких при рентгенографии или КТ (с наличием либо отсутствием грибкового шара), а также хронического кашля или кровохарканья, имеет чувствительность 85,6\% и специфичность 99,6\% [21]. Пациентам, находящимся в зоне риска возникновения ХАЛ, в том числе после перенесенного туберкулеза, показано последующее динамическое наблюдение с помощью низкодозовой КТ [10, 15].

\section{Саркоидоз}

Возникновение ХАЛ у пациентов с саркоидозом, по мнению D.W. Denning et al., характеризуется сложным клиническим течением и поздней диагностикой [28]. Однако согласно результатам ретроспективного одноцентрового исследования $(n=44)$ с группой контроля наличие ХАЛ может не оказывать существенного влияния на выживаемость пациентов с саркоидозом [29]. Основными причинами смерти являлись прогрессирование фонового заболевания и легочное кровотечение [29].

Неоднократно подчеркивалась клиническая и диагностическая сложность сочетания саркоидоза и ХАЛ [30, 31]. Лучевая диагностика данного состояния затруднена до периода формирования грибкового шара внутри гранулемы. На ранних стадиях патологического процесса большое значение имеют лабораторные методы диагностики ХАЛ [28].

Основными выявляемыми клинико-лучевыми формами ХАЛ в сочетании с саркоидозом в исследовании Y. Uzunhan et al. [29] являлись простая аспергиллома (31\%), кавитарный (27\%) и фиброзирующий (15\%) аспергиллез. По сравнению с другими фоновыми заболеваниями выявлен более высокий процент фиброзных изменений (рис. 3). 

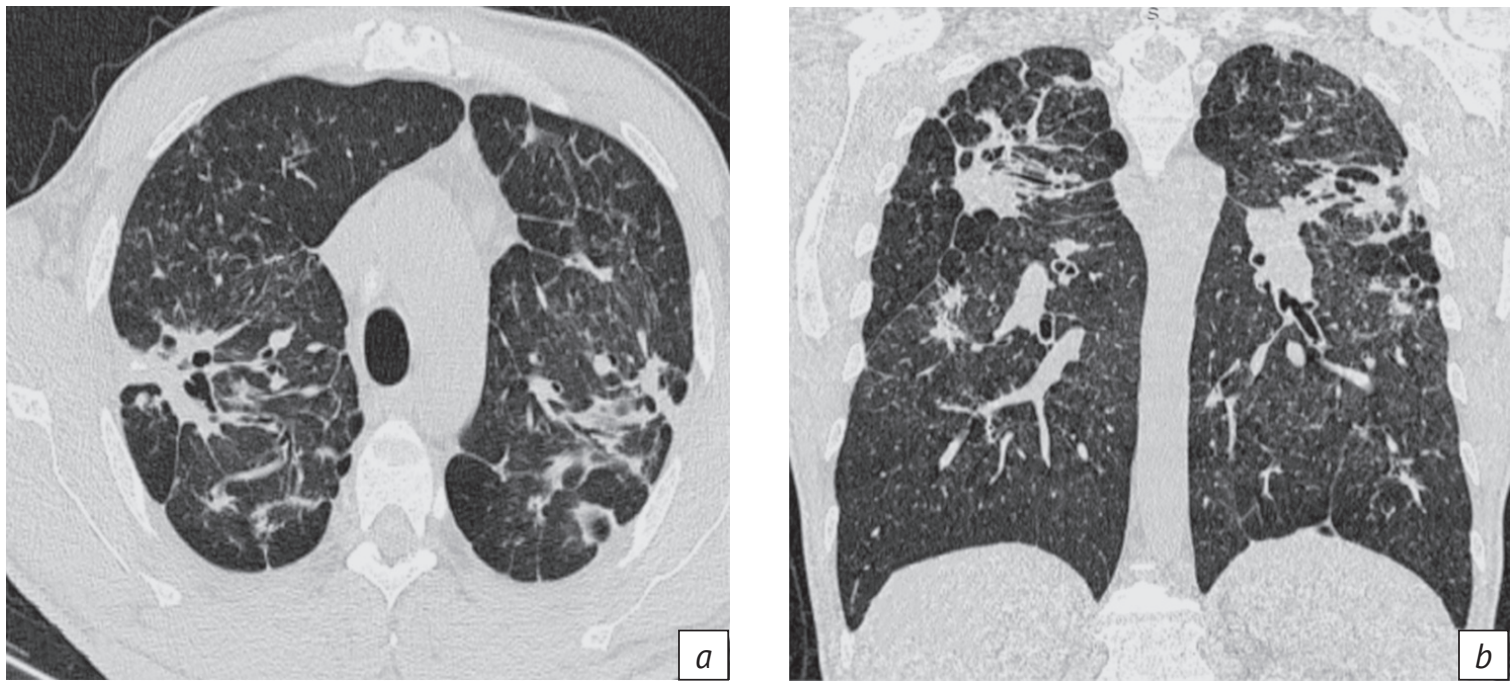

Puc. 3. Компьютерные томограммы пациента М., 30 лет: легочное окно, аксиальная (a) и корональная (b) проекции. Гистологически верифицированная легочная форма саркоидоза, осложненная ХАЛ. Длительный прием глюкокортикостероидов: двусторонние участки уплотнения легочной ткани, локализующиеся в верхних и прикорневых отделах, с формированием полостей и перифокального фиброза

Fig. 3. Computed tomography scans in male patient M. aged 30 years: pulmonary window, axial $(a)$ and coronal $(b)$ projections. Histologically verified pulmonary sarcoidosis complicated by CPA. Long-term use of glucocorticosteroids: bilateral lung tissue compaction areas located in the upper and basal segments with the formation of cavities and perifocal fibrosis

\section{Нетуберкулезный микобактериоз}

Нетуберкулезный микобактериоз (НTM) является установленным фактором риска развития ХАЛ, при этом во всех публикациях подчеркивается сложность диагностики сочетания этих инфекционных процессов [15, 32, 33].

В результате ретроспективного анализа данных 378 пациентов с НТМ частота развития ХАЛ составила 9,8\% [30]. Установлено, что образование полостей при нетуберкулезной микобактериальной инфекции связано со значительным риском развития ХАЛ $(p<0,001)$ [30].

Лучевая картина ХАЛ на фоне НТМ зачастую неспецифична. Наиболее распространенными признаками являются бронхоэктазии, очаги, фиброзно-кавернозные изменения [31]. У большинства больных специфических изменений на КТ не выявлено.

В крупном когортном исследовании $(n=566)$ пациентов с НМТ ХАЛ диагностирован у 41 больного $(7,2 \%)$ [32]. При этом статистически значимыми факторами риска являлись пожилой возраст, мужской пол, низкий индекс массы тела, ХОБЛ, использование системных стероидов, Mycobacterium abscessus complex в качестве этиологического возбудителя и фиброзно-полостная форма нетуберкулезного микобактериоза, выявляемая на КТ легких. Уровень летальности в группе пациентов с сочетанием нетуберкулезного микобактериоза и ХАЛ был выше, чем в группе контроля (19,5\% и 1,7\% соответственно, $p<0,001)$.

\section{Другие фоновые заболевания и клинические проявления ХАЛ}

Возможно развитие ХАЛ на фоне муковисцидоза с частотой до 4,2\% [33]. Отдельные сообщения свидетельствуют о возникновении ХАЛ у людей с профессиональными легочными заболеваниями [34], ревматологическими заболеваниями (в частности при анкилозирующем спондилите) [35], деструктивной и инфаркт-пневмонией [36].

ХАЛ является осложнением лобэктомии по поводу рака легкого, при этом частота развития микотической инфекции составила 2,3\% через 5 лет и 7,9\% через 10 лет [37]. Статистически значимыми факторами риска являются мужской пол, курение, ХОБЛ, идиопатический легочный фиброз.

Клинические проявления ХАЛ неспецифичны и складываются из симптомов фонового заболевания легких и системных проявлений. Практически во всех публикациях в качестве симптомов ХАЛ отмечают потерю веса, кашель с отделением мокроты, кровохарканье различной степени, боли в грудной клетке. Повышение температуры нехарактерно.

\section{Основные лучевые варианты ХАЛ}

\section{Простая одиночная аспергиллома}

Наиболее типичная форма ХАЛ - простая одиночная аспергиллома, или грибной шар, представляющий собой разрастающийся в полостях легких 

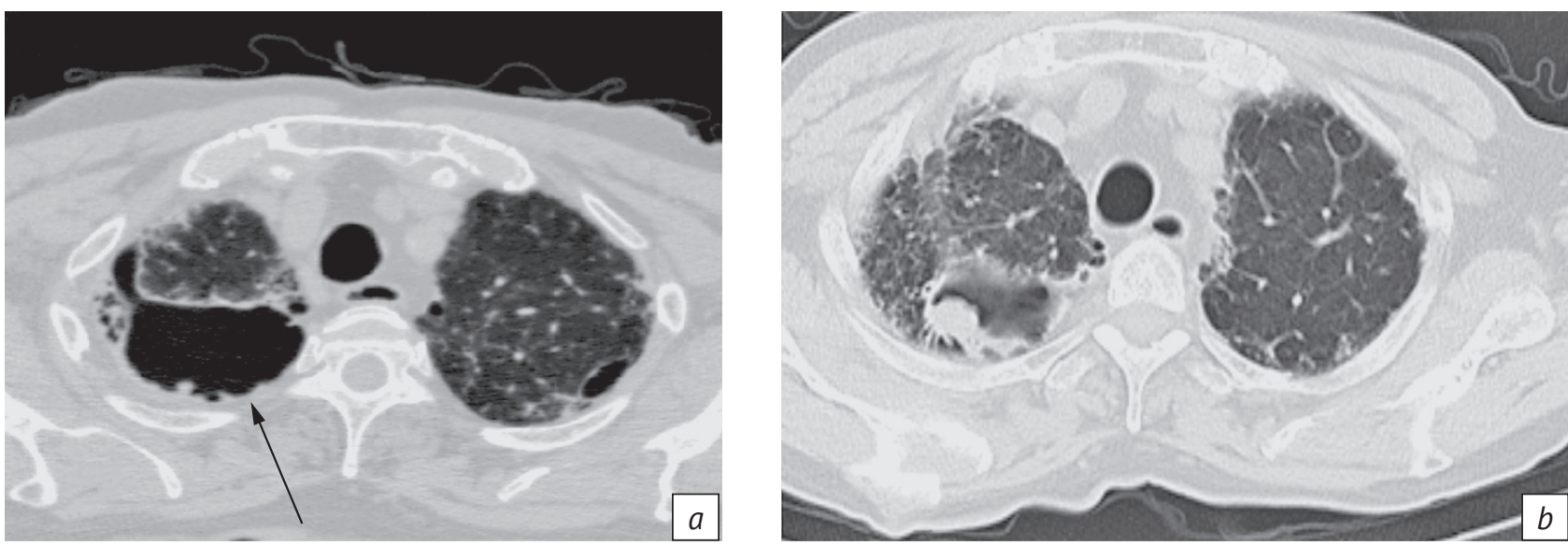

Puc. 4. Компьютерные томограммы органов грудной клетки пациента Д., 77 лет: легочное окно, аксиальная проекция. Основное заболевание - идиопатический легочный фиброз. Жалобы: одышка, кашель с мокротой слизисто-гнойного характера. Данных за туберкулез легких не получено. При первичном исследовании (a) в верхней доле правого легкого определяется полость с неровным внутренним контуром (стрелка). При обследовании через 3 мес (b) внутри полости сформировался грибковый шар. По результатам анализа крови IgG Aspergillus fumigatus 1:3200 (норма 1:100). Назначена антимикотическая терапия

Fig. 4. Chest computed tomography scans in male patient D. aged 77 years: pulmonary window, axial projection. The underlying disease is idiopathic pulmonary fibrosis. Complaints: dyspnea, cough with mucopurulent sputum. There is no evidence for pulmonary tuberculosis. Initial examination (a) has identified a cavity with an uneven inner contour (arrow) in the right upper lobe. Examination after 3 months $(b)$ has revealed a fungal ball formed inside the cavity. A blood test has shown Aspergillus fumigatusspecific IgG 1:3200 (normal value, 1:100); antifungal therapy is prescribed

мицелий Aspergillus spp. в совокупности с клеточным детритом, фибрином и т.д. [15, 38]. К настоящему времени этой форме аспергиллеза легких посвящено наибольшее число научных работ.

Перед формированием аспергилломы возможно увидеть плащевидный рост гриба внутри полости, выявляемый в виде неровности ее внутренней стенки при КТ легких [1] (рис. 4).

Простая одиночная аспергиллома на КТ характеризуется наличием внутриполостного содержимого, отделенного от стенки полости прослойкой воздуха, что в совокупности и формирует симптом полумесяца, или воздушного серпа. Однако этот симптом не является полностью специфичным и встречается при многих других заболеваниях как инфекционной, так и неинфекционной природы [39-42].

К одному из таких заболеваний, имитирующих аспергиллому на КТ, относится псевдоаневризма легочной артерии, или аневризма Расмуссена, представляющая собой редкое осложнение туберкулеза $[41,43]$. Как и при ХАЛ, при аневризме Расмуссена может развиваться угрожающее жизни легочное кровотечение [41], что значительно затрудняет дифференциальную диагностику данных состояний. В таких случаях показано внутривенное введение рентгенконтрастного препарата и полипозиционное сканирование.

Развитие абсцесса или гангрены легкого также сопровождается симптомом воздушного полумесяца. При этом в полости скапливаются не- кротические массы. Тяжесть клинической картины и динамика лучевой картины помогает дифференцировать абсцесс от других заболеваний, сопровождающихся внутриполостными массами.

Схожей с аспергилломой лучевой картиной могут обладать полостная форма аденокарциномы [42], метастазы [43], инфаркт-пневмония с зоной распада [44].

Изменения в легких, выявляемые при КТ-исследовании и имитирующие аспергиллому, могут наблюдаться при других редких инфекционных заболеваниях, таких как кокцидиоидомикоз, нокардиоз, актиномикоз, эхинококковые кисты легких $[41,45]$.

Для одиночной аспергилломы характерно отсутствие или слабая выраженность клинических симптомов. КТ является ведущим инструментальным методом диагностики.

В дополнение к грибковому субстрату на изображениях грудной клетки могут наблюдаться другие рентгенологические признаки, такие как утолщение паракостальной плевры и участки консолидации $[46,47]$. Несмотря на то что лучевые признаки достаточно показательны для простой одиночной аспергилломы, для точного диагноза требуется микробиологическое подтверждение.

Эндобронхиальная аспергиллома - малоизученное и редкое проявление хронического аспергиллеза легких, имитирующее на КТ эндобронхиальный рак легкого и характеризующееся ростом Aspergillus spp. в просвете бронха [48]. По данным 

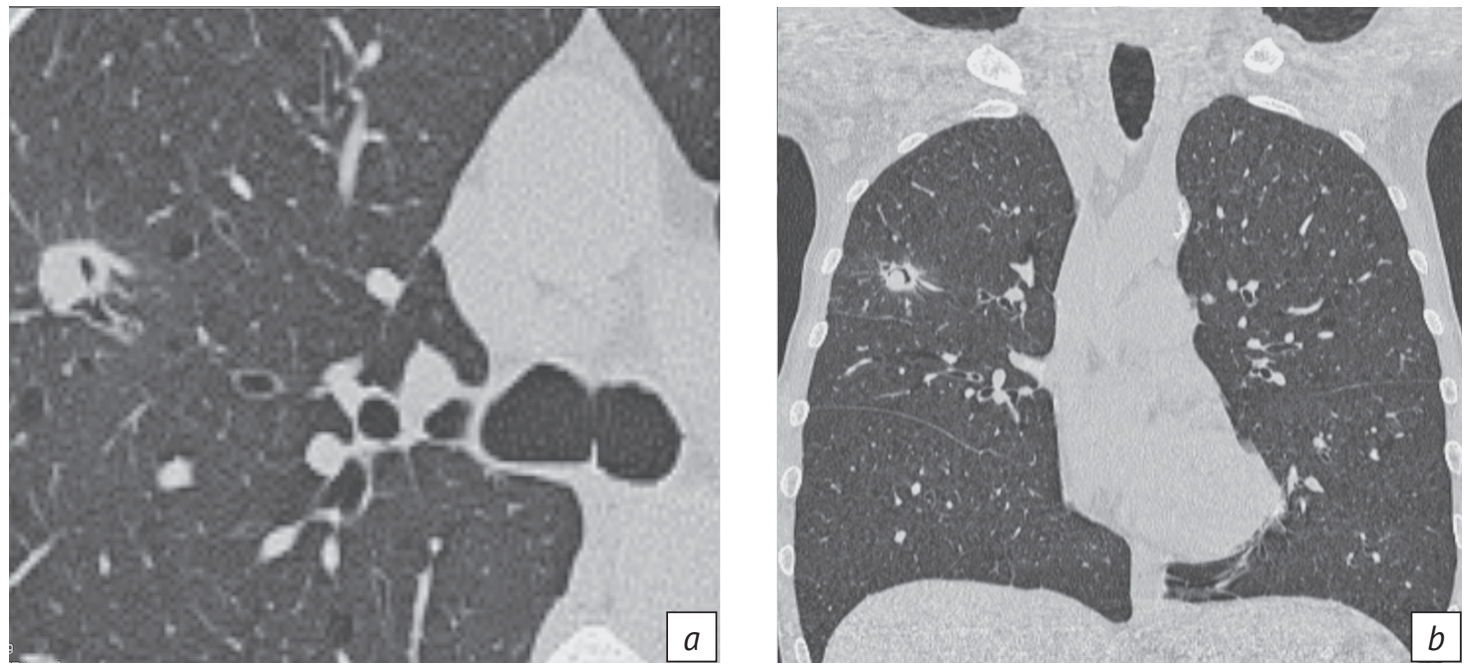

Puc. 5. Компьютерные томограммы пациента И., 62 года: легочное окно, аксиальная (a) и корональная (b) проекции: просвет бронха третьего сегмента верхней доли правого легкого локально расширен, с наличием мягкотканного образования, в окружающей легочной ткани зона пониженной пневматизации. Изменения в легких выявлены при плановой флюорографии. Лабораторные маркеры ХАЛ (серологические, микробиологические) дали отрицательный результат. Диагноз подтвержден при гистологическом исследовании постоперационного материала

Fig. 5. Computed tomography scans in male patient I. aged 62 years: pulmonary window, axial $(a)$ and coronal $(b)$ projections: the bronchial lumen in the third segment of the right upper lobe is locally expanded, with a soft tissue mass; there is a zone of reduced pneumatization in the surrounding lung tissue. Lung changes have been detected during routine fluorography. The laboratory (serological, microbiological) CPA markers have yielded a negative result. The diagnosis has been confirmed by histological examination of postoperative materials

S. Jiang et al. [49], а также F.Z. Mrabet et al. [50], возможно сочетание эндобронхиальной аспергилломы и злокачественных новообразований.

Как правило, эндобронхиальные аспергилломы являются случайной находкой при выполнении КТ или бронхоскопии (рис. 5). Однако в некоторых случаях данная патология может вызвать кровохарканье и нарушение вентиляции легочной паренхимы. В ретроспективном исследовании [51] сообщается о 16 пациентах с гистологически подтвержденным диагнозом эндобронхиальной аспергилломы. При этом только 3 больных имели предположительный диагноз грибкового поражения по данным КТ.

Основной вывод этих публикаций заключается в том, что аспергиллез необходимо рассматривать при проведении дифференциальной диагностики с другими эндобронхиальными поражениями.

\section{Хронический полостной (кавитарный) аспергиллез}

Хронический кавитарный аспергиллез, ранее называвшийся сложной аспергилломой, представляет собой несколько полостей, которые могут содержать грибной шар.

Неравномерно утолщенная стенка полости, содержащей грибной шар, морфологически состоит из фиброзной ткани с некрозом внутреннего слоя [46]. Стенка полости имеет активное кровоснабжение, что обусловливает склонность к легочному кровотечению и кровохарканью. В ретроспективно изученной серии из 60 пациентов, оперированных по поводу ХАЛ, полостной аспергиллез легких составил наибольшую долю случаев $(46,7 \%)$ [52].

\section{Хронический фиброзирующий аспергиллез}

Хронический фиброзирующий аспергиллез (ХФА), как правило, является конечной фазой нелеченого хронического кавернозного аспергиллеза с исходом в распространенный легочный фиброз [1]. Литературных данных по данной форме ХАЛ пока недостаточно, информация ограничена описанием небольших серий клинических наблюдений. Анализ компьютерных томограмм 11 пациентов Национального центра аспергиллеза Великобритании с ХФА показал выраженное или тотальное поражение легочной паренхимы с развитием симптома разрушенного легкого. При этом фиброз может как присутствовать в начале заболевания, так и развиваться в течение длительного времени [53].

В исследовании M. Sawahata et al., посвященном саркоидозу с прогрессирующим легочным фиброзом, хронический аспергиллез рентгенологически выявлен у 4 из 10 пациентов [54]. При этом имела место легочная гипертензия и выраженная дыхательная недостаточность. 


\section{Нодулярный аспергиллез}

Узловые образования в легких без формирования полости и грибкового шара являются малоизученным ранним проявлением ХАЛ [1, 55]. Нодулярный аспергиллез - наиболее сложная форма ХАЛ в плане дифференциальной диагностики с другими периферическими легочными новообразованиями. Большинство авторов отмечают, что дифференциальная диагностика нодулярного аспергиллеза по клиническим и рентгенологическим данным не всегда возможна $[1,56]$.

Современные публикации подтверждают, что позитронно-эмиссионная томография в сочетании с КТ с радиофармпрепаратом в диагностике хронического нодулярного аспергиллеза не является специфичным исследованием, так как аспергиллезные узлы могут активно накапливать ${ }^{18} \mathrm{~F}$-фтордезоксиглюкозу, что может создавать ложное представление о наличии опухоли $[45,57-59]$.

Описаны отдельные наблюдения множественных аспергиллезных узловых образований у пациентов с ревматоидным артритом $[13,60]$, а также случаи сходства нодулярной формы ХАЛ с метастатическими инфильтратами [43].

B исследовании N. Kang et al. сообщается о выявлении аспергиллезных узловых образований у 80 пациентов [55]. При этом лучистость контуров и кальцификация наблюдались у $20 \%$ и $39 \%$ больных соответственно. Положительный титр IgG Aspergillus обнаружен в 42\% случаев.

E.G. Muldoon et al. описывают узловые образования в легких без признаков полости у 33 человек [56]. В большинстве случаев (60\%) солидные образования локализовались в верхних долях, у 64\% пациентов выявлены множественные образования. Хронический нодулярный аспергиллез гистологически верифицирован у 7 из 13 больных, повышение уровня специфического IgG к Aspergillus spp. - у 4.

Таким образом, можно говорить об ограниченной информативности лабораторных тестов при данной форме ХАЛ.

Пациентам с одиночными узловыми образованиями в легких показано хирургическое лечение с последующей верификацией диагноза и назначением антимикотической терапии при подтверждении ХАЛ.

\section{Роль КТ в оценке прогрессирования ХАЛ и мониторинге лечения}

Согласно клиническим рекомендациям [1] пациентам с ХАЛ при невозможности хирургического лечения показана пероральная антимикотическая терапия в течение длительного времени. В результате годового наблюдения 206 больных установ- лено, что препараты на основе азолов умеренно эффективны в лечении ХАЛ, особенно если они назначаются в течение 12 мес [57]. Однако оптимальная продолжительность антимикотической терапии определяется особенностями каждого конкретного случая.

Для оценки эффективности лечения в клинических испытаниях антимикотических средств наряду с клиническими, микологическими и серологическими методами применяли КТ. Однако лучевые стандарты мониторинга лечения и критериев рецидива ХАЛ не установлены.

Немногочисленные работы посвящены КТоценке ответа на лечение хронического аспергиллеза легких. C. Godet et al. отмечали достоверную связь между сокращением размеров грибкового шара, уменьшением толщины стенки полости или плевры на КТ и клиническим улучшением [58].

Существуют сложности с понятием рецидива ХАЛ [59]: наличие фонового заболевания с возможностью обострения или присоединения бактериальной инфекции значительно затрудняют диагностику прогрессирования ХАЛ.

Данные исследования 102 пациентов, принимавших антимикотические препараты со средней продолжительностью 19 мес показали, что двусторонние изменения в легких и наличие аспергилломы являются статистически значимыми факторами риска рецидива ХАЛ, при этом длительная терапия (24 мес и более) снижает вероятность рецидива заболевания [59].

Рецидив может произойти даже после хирургической резекции очага ХАЛ. В ретроспективном исследовании 30 пациентов, перенесших операцию по поводу ХАЛ, он был отмечен в 8 случаях (26\%), что в будущем явилось неблагоприятным прогностическим признаком [61].

Кровохарканье и легочное кровотечение являются одним из наиболее грозных осложнений ХАЛ [62]. В группе из 60 больных с различными клинико-рентгенологическими формами ХАЛ частота кровохарканья составила 70\% [52]. Сообщения о связи размеров и структуры внутриполостного компонента при ХАЛ и развитием клинически значимого кровотечения противоречивы. В исследовании Т. Ando et al. [63] у 41 пациента ХАЛ осложнился легочным кровотечением. Наблюдалась высокая $(51,2 \%)$ частота рецидива кровотечения после эмболизации бронхиальных артерий в течение 24 мес. При этом достоверной связи между развитием легочного кровотечения и характеристиками КТ (толщина стенки полости, толщина плевры, одно- или двустороннее поражение, диаметр полостей, наличие или отсутствие грибкового шара) не отмечено ( $p>0,05)$. 
Напротив, в работе H. Sato et al. [64] описан струпоподобный признак внутри полости, патоморфологически соответствующий фиброзно-гнойной массе или сгустку крови. Пациенты с выявленным струпоподобным симптомом достоверно отличались от контрольной группы: кровохарканье у данной группы больных развилось в среднем в течение 12 дней после выполнения КТ. При этом достоверной корреляции между размерами полостей и грибкового шара и легочным кровотечением не выявлено.

\section{Заключение}

Приведенные данные свидетельствуют о необходимости комплексного подхода в диагностике ХАЛ, заключающегося в сочетании клинических, лабораторных и инструментальных методов исследования. Знание факторов риска, основных лучевых проявлений ХАЛ и его осложнений позволит повысить эффективность диагностики ХАЛ, вовремя начать адекватную терапию, осуществить качественный мониторинг лечения и улучшить прогноз заболевания.

\section{Литература [References]}

1. Denning DW, Cadranel J, Beigelman-Aubry C, et al. Chronic pulmonary aspergillosis: rationale and clinical guidelines for diagnosis and management. Eur Respir J. 2016; 47(1): 45-68. https://doi.org/10.1183/13993003.00583-2015.

2. Klimko N, Kozlova Y, Khostelidi S, et al. The burden of serious fungal diseases in Russia. Mycoses. 2015; 58(5): 58-62. https://doi.org/10.1111/myc.12388.

3. Osmanov A, Denning DW. Burden of serious fungal infections in Ukraine. Mycoses. 2015; 58(5): 94-100. https://doi.org/10.1111/myc.12409.

4. Bobokhojaev OI, Osmanov A, Aliev SP, et al. The burden of serious fungal infections in Tajikistan.J Fungi (Basel). 2019; 5(3): 68. https://doi.org/10.3390/jof5030068.

5. Lowes D, Al-Shair K, Newton PJ, et al. Predictors of mortality in chronic pulmonary aspergillosis. Eur Respir J. 2017; 49(2): 1601062. https://doi.org/10.1183/13993003.01062-2016.

6. Kosmidis C, Muldoon EG. Challenges in the management of chronic pulmonary aspergillosis. Med Mycol. 2017; 55(1): 63-8. https://doi.org/10.1093/mmy/myw119.

7. Laursen CB, Davidsen JR, Van Acker L, et al. CPAnet Registry An International Chronic Pulmonary Aspergillosis Registry. J Fungi (Basel). 2020; 6(3): 96. https://doi.org/10.3390/jof6030096.

8. Готман Л.Н., Юдин А.Л., Яцык Г.А. Ранние компьютерно-томографические признаки аспергиллеза легких при миелотоксическом агранулоцитозе. Медицинская визуализация. 2008; 3: 78-83.

[Gotman LN, Yudin AL, Yatsyk GA. The early CT-signs of pulmonary aspergillosis at myelotoxic agranulocytosis. Medical Visualization. 2008; 3: 78-83 (in Russ.).]

9. Трофимова Т.Н., Климко Н.Н., Самохвалова М.В. Лучевая диагностика инвазивного аспергиллеза легких. Медицинская визуализация. 2008; 2: 44-51.

[Trofimova TN, Klimko NN, Samokhvalova MV. Diagnostic imaging of invasive pulmonary aspergillosis. Medical Visualization. 2008; 2: 44-51 (in Russ.).]

10. Maghrabi F, Denning DW. The management of chronic pulmonary aspergillosis: The UK National Aspergillosis Centre approach. Curr Fungal Infect Rep. 2017; 11(4): 242-51. https://doi.org/10.1007/s12281-017-0304-7.

11. Paulussen C, Hallsworth JE, Alvarez-Perez S, et al. Ecology of aspergillosis: insights into the pathogenic potency of Aspergillus fumigatus and some other Aspergillus species. Microb Biotechnol. 2017; 10(2): 296-322. https://doi.org/10.1111/1751-7915.12367.

12. Denning DW, Chakrabarti A. Pulmonary and sinus fungal diseases in non-immunocompromised patients. Lancet Infect Dis. 2017; 17(11): 357-66. https://doi.org/10.1016/S1473-3099(17)30309-2.
13. Moazam S, Denning DW. Aspergillus nodules in chronic granulomatous disease attributable to Aspergillus ochraceus. Med Mycol Case Rep. 2017; 17: 31-3. https://doi.org/10.1016/j.mmcr.2017.06.004.

14. Larkin PM, Multani A, Beaird OE, et al. Collaborative tale of diagnosing and treating chronic pulmonary aspergillosis, from the perspectives of clinical microbiologists, surgical pathologists, and infectious disease clinicians. J Fungi (Basel). 2020; 6(3): 106. https://doi.org/10.3390/jof6030106.

15. Kosmidis C, Denning DW. The clinical spectrum of pulmonary aspergillosis. Thorax. 2015; 70(3): 270-7. https://doi.org/10.1136/thoraxjnl-2014-206291.

16. Sehgal IS, Choudhary H, Dhooria S, et al. Diagnostic cut-off of Aspergillus fumigatus-specific IgG in the diagnosis of chronic pulmonary aspergillosis. Mycoses. 2018; 61(10): 770-6. https://doi.org/10.1111/myc.12815.

17. Li H, Rui Y, Zhou W, et al. Role of the Aspergillus-specific $\mathrm{IgG}$ and IgM test in the diagnosis and follow-up of chronic pulmonary aspergillosis. Front Microbiol. 2019; 10: 1438. https://doi.org/10.3389/fmicb.2019.01438.

18. Sehgal IS, Dhooria S, Choudhary H, et al. Efficiency of a fumigatus-specific $\lg \mathrm{G}$ and galactomannan testing in the diagnosis of simple aspergilloma. Mycoses. 2019; 62(12): 1108-15. https://doi.org/10.1111/myc.12987.

19. Page ID, Richardson M, Denning DW. Antibody testing in aspergillosis - quo vadis? Med Mycol. 2015; 53(5): 417-39. https://doi.org/10.1093/mmy/myv020.

20. Sehgal IS, Dhooria S, Choudhary H, et al. Utility of serum and bronchoalveolar lavage fluid galactomannan in diagnosis of chronic pulmonary aspergillosis. J Clin Microbiol. 2019; 57(3): e01821-18. https://doi.org/10.1128/JCM.01821-18.

20. Page ID, Richardson MD, Denning DW. Comparison of six Aspergillus-specific $\lg G$ assays for the diagnosis of chronic pulmonary aspergillosis (CPA).J Infect. 2016; 72(2): 240-9. https://doi.org/10.1016/j.jinf.2015.11.003.

21. Page ID, Byanyima R, Hosmane S, et al. Chronic pulmonary aspergillosis commonly complicates treated pulmonary tuberculosis with residual cavitation. Eur Respir J. 2019; 53(3): 1801184. https://doi.org/10.1183/13993003.01184-2018.

22. Sehgal IS, Dhooria S, Muthu V, et al. An overview of the available treatments for chronic cavitary pulmonary aspergillosis. Expert Rev Respir Med. 2020; 14(7): 715-27. https://doi.org/10.1080/17476348.2020.1750956.

23. Méchaï F, Fock-Yee C, Bouvry D, et al. Pulmonary tuberculosis: radiological evolution of broncho-pulmonary lesions at the end of treatment. Rev Mal Respir. 2019; 36(1): 22-30. https://doi.org/10.1016/j.rmr.2018.08.005. 
24. Khan R, Malik NI, Razaque A. Imaging of pulmonary posttuberculosis sequelae. Pak J Med Sci. 2020; 36(1): S75-82. https://doi.org/10.12669/pjms.36.ICON-Suppl.1722.

25. Евсеев П.Ю., Белоказанцева В., Кондакова М.Н. и др. Хронический аспергиллез у пациентов с деструктивным туберкулезом легких. Проблемы медицинской микологии. 2018; 20(4): 24-6.

[Evseev PY, Belokazanceva V, Kondakova MN, et al. Chronic aspergillosis in adult patients with chronic destructive pulmonary tuberculosis. Problems in Medical Mycology. 2018; 20(4): 24-6 (in Russ.).]

26. Denning DW, Page ID, Chakaya J, et al. Case definition of chronic pulmonary aspergillosis in resource-constrained settings. Emerg Infect Dis. 2018; 24(8): e171312. https://doi.org/10.3201/eid2408.171312.

27. Naito M, Kurahara Y, Yoshida S, et al. Prognosis of chronic pulmonary aspergillosis in patients with pulmonary nontuberculous mycobacterial disease. Respir Investig. 2018; 56(4): 326-31. https://doi.org/10.1016/j.resinv.2018.04.002.

28. Denning DW. Sarcoidosis and aspergillosis: a tough combination. Eur Respir J. 2017; 49(6): 1700574. https://doi.org/10.1183/13993003.00574-2017.

29. Uzunhan Y, Nunes H, Jeny F, et al. Chronic pulmonary aspergillosis complicating sarcoidosis. Eur Respir J. 2017; 49(6): 1602396. https://doi.org/10.1183/13993003.02396-2016.

30. Fujiuchi S, Fujita Y, Suzuki H, et al. Cavitary formation in nontuberculous mycobacterial infection is the significant risk for development to chronic pulmonary aspergillosis. Eur Respir J. 2014; 44(Suppl 58): P2491.

31. Takeda K, Imamura Y, Takazono T, et al. The risk factors for developing of chronic pulmonary aspergillosis in nontuberculous mycobacteria patients and clinical characteristics and outcomes in chronic pulmonary aspergillosis patients coinfected with nontuberculous mycobacteria. Med Mycol. 2016; 54(2): 120-7. https://doi.org/10.1093/mmy/myv093.

32. Jhun BW, Jung WJ, Hwang NY, et al. Risk factors for the development of chronic pulmonary aspergillosis in patients with nontuberculous mycobacterial lung disease. PLoS One. 2017; 12(11): e0188716. https://doi.org/10.1371/journal.pone.0188716.

33. Козлова Я.И., Борзова Ю.В., Шадривова О.В. и др. Аспергиллез легких у больных муковисцидозом в Российской Федерации. Журнал инфектологии. 2018; 10(2): 48-54. https://doi.org/10.22625/2072-6732-2018-10-2-48-54. [Kozlova Yal, Borzova YuV, Shadrivova OV, et al. Pulmonary aspergillosis in patients with cystic fibrosis in Russian Federation. Journal of Infectology. 2018; 10 (2): 48-54 (in Russ.). https://doi.org/10.22625/2072-6732-2018-10-2-48-54.]

34. Шпагина Л.А., Паначева Л.А., Куделя Л.М. и др. Аспергиллез легких среди рабочих высокого профессионального риска. Пульмонология. 2014; 1: 113-5. https://doi.org/10.18093/0869-0189-2014-0-1-113-115. [Shpagina LA, Panacheva LA, Kudelya LM, et al. Pulmonary aspergillosis in workers with high occupational risk. Pulmonologiya. 2014; 1: 113-5 (in Russ.). https://doi.org/10.18093/0869-0189-2014-0-1-113-115.]

35. Трофименко И.Н., Нашатырева М.С., Черняк Б.А. Поражение легких при анкилозирующем спондилите. Пульмонология. 2017; 27(1): 97-102. https://doi.org/10.18093/0869-0189-2017-27-1-97-102.
[Trofimenko IN, Nashatyreva MS, Chernyak BA. Pulmonary involvement in ankylosing spondylitis. Pulmonologiya. 2017; 27(1): 97-102 (in Russ.). https://doi.org/10.18093/0869-0189-2017-27-1-97-102.]

36. Desai SR, Hedayati V, Patel K, Hansell DM. Chronic aspergillosis of the lungs: unravelling the terminology and radiology. Eur Radiol. 2015; 25(10): 3100-7. https://doi.org/10.1007/s00330-015-3690-7.

37. Tamura A, Suzuki J, Fukami T, et al. Chronic pulmonary aspergillosis as a sequel to lobectomy for lung cancer. Interact Cardiovasc Thorac Surg. 2015; 21(5): 650-6. https://doi.org/10.1093/icvts/ivv239.

38. Panse $P$, Smith $M$, Cummings $K$, et al. The many faces of pulmonary aspergillosis: Imaging findings with pathologic correlation. Radiol Infect Dis. 2016; 3(4): 192-200. https://doi.org/10.1016/j.jrid.2016.10.002.

39. Sevilha JB, Rodrigues RS, Barreto MM, et al. Infectious and noninfectious diseases causing the air crescent sign: a state-ofthe-art review. Lung. 2018; 196(1): 1-10. https://doi.org/10.1007/s00408-017-0069-3.

40. Gazzoni FF, Severo LC, Marchiori E, et al. Pulmonary diseases with imaging findings mimicking aspergilloma. Lung. 2014; 192(3): 347-57. https://doi.org/10.1007/s00408-014-9568-7.

41. Marchiori E, Hochhegger B, Zanetti G. Intracavitary nodule in active tuberculosis: differential diagnosis of aspergilloma. J Bras Pneumol. 2015; 41(6): 562-3. https://doi.org/10.1590/S1806-37562015000000211.

42. Taoka T, Shinohara T, Naruse K, Ogushi F. Cavitary lung cancer with cartilage tissues in the wall mimicking aspergilloma. Thorax. 2017; 72(4): 383-4. https://doi.org/10.1136/thoraxjnl-2016-209437.

43. Demirtaş H, Çelik AO, Kayan M, et al. Aspergilloma mimicking metastasis in a case with laryngeal carcinoma. Med Arch. 2016; 70(1): 76-8. https://doi.org/10.5455/medarh.2016.70.76-78.

44. Tunney R, Rodger K, Denning DW, Kosmidis C. Chronic pulmonary aspergillosis following pulmonary embolism. Med Mycol Case Rep. 2019; 23: 20-2. https://doi.org/10.1016/j.mmcr.2018.11.002.

45. Higashi Y, Nakamura S, Ashizawa N, et al. Pulmonary actinomycosis mimicking pulmonary aspergilloma and a brief review of the literature. Intern Med. 2017; 56(4): 449-53. https://doi.org/10.2169/internalmedicine.56.7620.

46. Ando T, Tochigi N, Gocho K, et al. pathophysiological implication of computed tomography images of chronic pulmonary aspergillosis. Jpn J Infect Dis. 2016; 69(2): 118-26. https://doi.org/10.7883/yoken.JJID.2015.028.

47. Desai SR, Hedayati V, Patel K, Hansell DM. Chronic aspergillosis of the lungs: unravelling the terminology and radiology. Eur Radiol. 2015; 25(10): 3100-7. https://doi.org/10.1007/s00330-015-3690-7.

48. Araújo D, Figueiredo M, Monteiro P. Endobronchial aspergilloma: an unusual presentation of pulmonary aspergillosis. Rev Port Pneumol. 2016; 22(1): 61-2. https://doi.org/10.1016/j.rppnen.2015.07.003.

49. Jiang S, Jiang L, Shan F, et al. Two cases of endobronchial aspergilloma with lung cancer: a review the literature of endobronchial aspergilloma with underlying malignant lesions of the lung. Int J Clin Exp Med. 2015; 8(9): 17015-21.

50. Mrabet FZ, Soualhi M, Achrane J, et al. The tree that hides the forest: aspergillosis associated with bronchial carcinoma (about two cases). Pan Afr Med J. 2017; 28: 302. https://doi.org/10.11604/pamj.2017.28.302.11665. 
51. Huang D, Li B, Chu H, et al. Endobronchial aspergilloma: a case report and literature review. Exp Ther Med. 2017; 14(1): 547-54. https://doi.org/10.3892/etm.2017.4540.

52. He B, Wan C, Zhou W, et al. Clinical profile and surgical outcome for different typeof chronic pulmonary aspergillosis. Am J Transl Res. 2019; 11(6): 3671-9.

53. Kosmidis C, Newton P, Muldoon EG, Denning DW. Chronic fibrosing pulmonary aspergillosis: a cause of 'destroyed lung' syndrome. Infect Dis (Lond). 2017; 49(4): 296-301. https://doi.org/10.1080/23744235.2016.1232861.

54. Sawahata M, Johkoh T, Kawanobe T, et al. Computed tomography images of fibrotic pulmonary sarcoidosis leading to chronic respiratory failure.J Clin Med. 2020; 9(1): 142. https://doi.org/10.3390/jcm9010142.

55. Kang N, Park J, Jhun BW. Clinical characteristics and treatment outcomes of pathologically confirmed aspergillus nodules. J Clin Med. 2020; 9(7): 2185. https://doi.org/10.3390/jcm9072185.

56. Muldoon EG, Sharman A, Page I, et al. Aspergillus nodules; another presentation of chronic pulmonary aspergillosis. BMC Pulm Med. 2016; 16(1): 123. https://doi.org/10.1186/s12890-016-0276-3.

57. Bongomin F, Harris C, Hayes G, et al. Twelve-month clinical outcomes of 206 patients with chronic pulmonary aspergillosis. PLoS One. 2018; 13(4): e0193732. https://doi.org/10.1371/journal.pone.0193732.
58. Godet C, Laurent F, Bergeron A, et al. CT imaging assessment of response to treatment in chronic pulmonary aspergillosis. Chest. 2016; 150(1): 139-47. https://doi.org/10.1016/j.chest.2016.02.640.

59. Bongomin F, Otu A, Harris C, et al. Risk factors for relapse of chronic pulmonary aspergillosis after discontinuation of antifungal therapy. Clin Infect Pract. 2020; 5: 100017. https://doi.org/10.1016/j.clinpr.2020.100015.

60. Ito Y, Tanigawa M, Takemura T, et al. Chronic nodular pulmonary aspergillosis in a patient with rheumatoid arthritis. Intern Med. 2019; 58(7): 979-84. https://doi.org/10.2169/internalmedicine.1918-18.

61. Farid S, Mohamed S, Devbhandari M, et al. Results of surgery for chronic pulmonary aspergillosis, optimal antifungal therapy and proposed high risk factors for recurrence - a National Centre's experience.J Cardiothorac Surg. 2013; 8: 180. https:// doi.org/10.1186/1749-8090-8-180.

62. Kim TH, Koo HJ, Lim CM, et al. Risk factors of severe hemoptysis in patients with fungus ball.J Thorac Dis. 2019; 11(10): 4249-57. https://doi.org/10.21037/jtd.2019.09.52.

63. Ando T, Kawashima M, Masuda K, et al. Exacerbation of chronic pulmonary aspergillosis was associated with a high rebleeding rate after bronchial artery embolization. Respir Investig. 2019; 57(3): 260-7. https://doi.org/10.1016/j.resinv.2018.12.009.

64. Sato H, Okada F, Matsumoto S, et al. The scab-like sign: a CT finding indicative of haemoptysis in patients with chronic pulmonary aspergillosis? 2018; 28(10): 4053-61. https://doi.org/10.1007/s00330-018-5434-y. 